International Institute for Applied Systems Analysis - A-2361 Laxenburg - Austria Tel: +432236807 • Fax: +43223671313 • E-mail: info@iiasa.ac.at •Web: www.iiasa.ac.at

INTERIM REPORT IR-98-104 /December

\title{
Restricting Weights in Value Efficiency Analysis
}

Merja Halme(mhalme@hkkk.fi)

Pekka Korhonen(korhonen@iiasa.ac.at)

\section{Approved by}

Gordon Mac Donald (macdon@ilasa .ac .at)

Director, IIASA

Interim Reports on work of the International Institute for Applied Systems Analysis receive only limited review. Views or opinions expressed herein do not necessarily represent those of the Institute, its National Member Organizations, or other organizations supporting the work. 


\section{Contents}

1. Introduction 1

2. Data Envelopment Analysis with Weights Restrictions 2

2.1 General DEA Model 2

2.2 DEA Model with Weights Restrictions 4

2.3 An Example - Efficiency Analysis of Municipal Dental Units 5

3. Setting restrictions on Multipliers in Value Efficiency Analysis 7

3.1 Value Efficiency Analysis $\quad 7$

$\begin{array}{ll}3.2 \text { Restricting Weights in Value Efficiency Analysis } & 10\end{array}$

3.3 An Example - Value Efficiency Analysis of Municipal Dental Units 12

4. Further Considerations 13

4.1 The set of weights, optimal for an efficient unit 13

$\begin{array}{ll}4.2 \text { Illustrative Example } & 14\end{array}$

5. Conclusion 16 


\section{Abstract}

In this paper, we consider the problem of incorporating additional preference information into Value Efficiency Analysis by using the "price" information of inputs and outputs. This is done to improve the accuracy of the estimation of the Value Efficiency Scores. Value Efficiency developed by Halme et al [1998] is an efficiency concept, which takes into account the decision maker's preferences. Value Efficiency Analysis is based on the assumption that an implicitly known value function reaches its maximum at the Most Preferred Solution on the efficient frontier. The Most Preferred solution is an input-output vector preferred to all other possible input-output vectors. The ultimate goal is to measure a need to improve (radially) the values of inputs and/or outputs to make them equally preferred to the Most Preferred Solution. Because we do not know the value function, we approximate the indifference curves of all possible value functions satisfying certain assumptions by their tangents at the Most Preferred Solution. The resulting cone of the tangents consists of points less or equally preferred tothe Most Preferred Solution. However, in addition to the Most Preferred Solution information about the "prices" of inputs and outputs may be available as well. We show how this information can be incorporated into the analysis and illustrate the approach by an example on the performance of municipal dental units in Finland.

Keywords: Efficiency Analysis, Data Envelopment Analysis, Value Efficiency, Value Function, Multipliers. 


\section{Acknowledgments}

The research was supported, in part, by grants from the Foundation of the Helsinki School of Economics and Business Administration and Academy of Finland. 


\section{About the Authors}

Merja Halme is a senior researcher at the Helsinki School of Economics and Business Administration.

Pekka Korhonen is Project Leader of the Decision Analysis and Support Project at IIASA, and also Professor of Statistics at the Department of Economics and Management Science, Helsinki School of Economics and Business Administration 


\title{
Restricting Weights in Value Efficiency Analysis
}

\author{
Merja Halme \\ Pekka Korhonen
}

\section{Introduction}

Data Envelopment Analysis (DEA) introduced by Charnes et al. [1978] has gained a wide range of applications measuring comparative efficiency. It is a non-parametric method based on linear programming. The productivities of units, specified as the ratio of the weighted sum of outputs and inputs, are compared with each other and efficient units are identified. The units can be analyzed together only if they use the same inputs and produce the same outputs with similar production technologies. In a DEA programme, optimal weights are defined for each unit's all inputs and outputs, i.e. the weights that are the most favorable for the unit.

It is considered an advantage of DEA that no preference information is needed that the weights are allowed total flexibility. However, many value judgment schemes have been proposed for several reasons. The first weights restrictions in DEA were put forward by Thompson et al. [1986]. Weights restrictions are the most straightforward way to incorporate preference information in a DEA analysis. It is often the case that some information on the relative importance or relative prices of the inputs and outputs is available. It would not be reasonable to exclude that from the analysis. The weights do not always have an interpretation as prices.

Outside of weights restrictions, preference information can be incorporated into DEA by target setting (e.g. Golany [1988] and Thanassoulis and Dyson [1992]), by adding unobserved (artificial) decision-making units (DMU) into the analysis (Thanassoulis et al. [1998]) or by Value Efficiency Analysis (Halme et al. [1998]). For reviews on value judgment schemes, see Allen et al. [1997] and Pedraja et al. [1997].

Value Efficiency Analysis (VEA) is an approach to incorporate value judgments in DEA via the most preferred solution (MPS), which is the input-output vector on the efficient frontier preferred by the decision maker (DM) to all other possible input-output vectors. To insert this information into efficiency analysis, requires a modification of the original model. The modification changes the efficient frontier. In that sense, the Value Efficiency Analysis is analogous to weights restrictions models. In Value Efficiency Analysis, the DM does not explicitly consider the weights. He/she only chooses the MPS among all the efficient (virtual) units. 
In this paper we propose the use of both kinds of preference information in the analysis: the most preferred input-output vector as well as information on weights of inputs and/or outputs, when it is reasonable. We may apply weights restrictions before or after Value Efficiency Analysis. In this paper we deal with both cases. The use of weights restrictions in the context of Value Efficiency Analysis can be interpreted as an aim to improve the precision of the approximation of Value Efficiency Scores.

To illustrate our approach, we present an example concerning the analysis of municipal dental units in Finland, where there are two kinds of labor inputs: better paid dentists and less paid other staff (in man years). The outputs are divided into two parts: patients under 18 years of age and other patients (that have been taken care of). The treatment of the latter group is more expensive. We demonstrate that we get very useful results by combining these two different ways to incorporate preference information.

The rest of this paper is organized as follows. Section 2 reviews the basic DEA-models and the traditional weights restriction approach. Section 3 provides an introduction to Value Efficiency Analysis and its use together with weights restrictions. Section 4 considers the properties and use of the set of weights optimal for the MPS. Section 5 concludes the paper.

\section{Data Envelopment Analysis with Weights Restrictions}

\subsection{General DEA Model}

Assume we have $n$ decision making units (DMU) each consuming $m$ inputs and producing $p$ outputs. Let $\mathbf{X} \in \mathfrak{R}_{+}^{m \times n}$ and $\mathbf{Y} \in \mathfrak{R}_{+}^{p \times n}$ be matrices, consisting of nonnegative elements, containing the observed input and output measures for the DMUs. We further assume that there are no duplicated units in the data set. We denote by $\boldsymbol{x}_{j}$ (the $\mathrm{j}^{\text {th }}$ column of $\mathbf{X}$ ) the vector of inputs consumed by $\mathrm{DMU}_{j}$, and by $x_{i j}$ the quantity of input $i$ consumed by $\mathrm{DMU}_{j}$. A similar notation is used for outputs. When it is not necessary to emphasize the different roles of inputs and outputs, we denote $\boldsymbol{u}=\left[\begin{array}{c}\boldsymbol{y} \\ -\boldsymbol{x}\end{array}\right]$ and $\mathbf{U}=\left[\begin{array}{r}\mathbf{Y} \\ -\mathbf{X}\end{array}\right] \cdot{ }^{1)}$ Furthermore, we denote $\mathbf{l}=[1, \ldots, 1]^{\mathrm{T}}$ and refer by $\boldsymbol{e}_{i}$ to the $i^{\text {th }}$ unit vector in $\Re^{\mathrm{n}}$.

We consider set $\mathrm{T}=\{\boldsymbol{u} \mid \boldsymbol{u}=\mathbf{U} \lambda, \lambda \in \Lambda\}$, where $\Lambda=\left\{\lambda \mid \lambda \in \mathfrak{R}_{+}^{n}\right.$ and $\left.\mathbf{A} \lambda \leq \boldsymbol{b}\right\}, \boldsymbol{e}_{i} \in$ $\Lambda, i=1, \ldots, n$. Further consider matrix $\mathbf{A} \in \mathfrak{R}^{k \times n}$ and vector $\boldsymbol{b} \in \mathfrak{R}^{k}$ which are used to specify the feasible values of $\lambda$-variables. All efficient DMUs lie on the efficient

\footnotetext{
${ }^{1)}$ Because the results concerning $\boldsymbol{u}$ and $\mathbf{U}$ are valid for $\left[\begin{array}{c}\boldsymbol{y} \\ \boldsymbol{x}\end{array}\right]$ and $\left[\begin{array}{l}\mathbf{Y} \\ \mathbf{X}\end{array}\right]$ as well, for simplicity, we often refer to $\boldsymbol{u}$ and $\mathbf{U}$, although we are factually interested in results concerning $\left[\begin{array}{l}\boldsymbol{y} \\ \boldsymbol{x}\end{array}\right]$ and $\left[\begin{array}{l}\mathbf{Y} \\ \mathbf{X}\end{array}\right]$.
} 
frontier, which is defined as a subset of points of set $\mathrm{T}$ satisfying the efficiency condition defined below:

Definition 1. Point $\boldsymbol{u}^{*} \in \mathrm{T}$ is efficient iff there does not exist another $\boldsymbol{u} \in \mathrm{T}$ such that $\boldsymbol{u}$ $\geq \boldsymbol{u}^{*}$, and $\boldsymbol{u} \neq \boldsymbol{u}^{*}$.

Definition 2. Point $\boldsymbol{u}^{*} \in \mathrm{T}$ is weakly efficient iff there does not exist another $\boldsymbol{u} \in \mathrm{T}$ such that $\boldsymbol{u}>\boldsymbol{u}^{*}$.

The purpose of DEA is to diagnose which of the existing units $\boldsymbol{u}_{j}=\mathrm{Ue}_{j}\left(\boldsymbol{u}_{j} \in \mathrm{T}, j=1\right.$, $2, \ldots, n)$ are efficient and how inefficient the rest of the units are. The original DEAmodels as introduced by Charnes et al. [1978] were constant returns to scale models, later on called CCR-models, i.e. set $\mathrm{T}$ was defined by setting $\Lambda=\left\{\lambda \mid \lambda \in \mathfrak{R}_{+}^{n}\right\}$. Later Banker, Charnes and Cooper [1984] developed the BCC models with variable returns to scale, i.e. $\Lambda=\left\{\lambda \mid \lambda \in \mathfrak{R}_{+}^{n}\right.$ and $\left.\boldsymbol{1}^{\mathrm{T}} \lambda=1\right\}$ The CCR and BCC-models are the most common model types in DEA. To unify the presentation we formulate a general model (for short, GEN) which includes CCR- and BCC-models as special cases. We would like to emphasize that subsequent considerations are valid for other standard DEA-models as well. Note that the original primal formulation in Charnes et al. [1978]) is currently in the DEA-literature (see, e.g. Charnes et al. [1994]) called the dual and vice versa.

\begin{tabular}{|c|c|}
\hline $\begin{array}{c}\text { General DEA Model } \\
\text { (Primal) }\end{array}$ & $\begin{array}{c}\text { General DEA Model } \\
\text { (Dual) }\end{array}$ \\
\hline $\max \quad \mathrm{Z}=\sigma+\varepsilon\left(\boldsymbol{1}^{T} \boldsymbol{s}^{+}+\boldsymbol{1}^{T} \boldsymbol{s}^{-}\right)$ & $\operatorname{Min} \quad W=v^{T} \boldsymbol{g}^{x}-\mu^{T} \boldsymbol{g}^{y}+\eta^{T} \boldsymbol{b}$ \\
\hline $\begin{array}{l}\text { s.t. } \\
\begin{array}{l}\mathbf{Y} \lambda-\sigma w^{y}-s^{+}=g^{y} \\
\mathbf{X} \lambda+\sigma w^{x}+s^{-}=g^{x} \\
\mathrm{~A} \lambda+\delta=b \\
\lambda, \delta, s^{-}, s^{+} \geq \boldsymbol{0} \\
\varepsilon>0, \text { ("Non-Archimedean") }\end{array}\end{array}$ & $\begin{array}{l}\text { s.t. } \\
\qquad \begin{array}{l}-\mu^{\mathrm{T}} \mathbf{Y}+v^{\mathrm{T}} \mathbf{X}+\eta^{\mathrm{T}} \mathbf{A}-\gamma^{T}=\boldsymbol{0}^{T} \\
\mu^{T} \boldsymbol{w}^{y}+v^{\mathrm{T}} \boldsymbol{w}^{x} \quad=1 \\
\mu, v \geq \varepsilon \boldsymbol{I} \\
\gamma, \eta \geq \boldsymbol{0} \\
\varepsilon>0, \text { ("Non-Archimedean") }\end{array}\end{array}$ \\
\hline
\end{tabular}

Vector $\boldsymbol{g}^{y}$ consists of general aspiration levels for outputs and $\boldsymbol{g}^{x}$ of general aspiration levels for inputs. Denote $\boldsymbol{g}=\left[\begin{array}{l}\boldsymbol{g}^{y} \\ \boldsymbol{g}^{x}\end{array}\right] \in \mathfrak{R}^{\mathrm{m}+\mathrm{p}}$. Vectors $\boldsymbol{w}^{y} \geq \boldsymbol{0}$ and $\boldsymbol{w}^{x} \geq \boldsymbol{0}$ with $\boldsymbol{w}=\left[\begin{array}{l}\boldsymbol{w}^{y} \\ \boldsymbol{w}^{x}\end{array}\right] \neq \boldsymbol{0}$ are the weighting vectors for outputs and inputs, respectively. By choosing the vectors $g$ and $\boldsymbol{w}$ in an appropriate way, we will get one of the original DEA-models. 
In DEA-models, $\boldsymbol{g}$ is one of the existing units $\left[\begin{array}{l}\boldsymbol{y}_{j} \\ \boldsymbol{x}_{j}\end{array}\right]=\left[\begin{array}{l}\mathbf{Y} \\ \mathbf{X}\end{array}\right] \mathbf{e}_{j}\left(\boldsymbol{u}_{j} \in \mathrm{T}, j=1,2, \ldots, \mathrm{n}\right)$. Vector $\boldsymbol{w}$ is either $\left[\begin{array}{c}\boldsymbol{y}_{j} \\ \boldsymbol{0}\end{array}\right],\left[\begin{array}{l}\boldsymbol{0} \\ \boldsymbol{x}_{j}\end{array}\right]$, or $\left[\begin{array}{l}\boldsymbol{y}_{j} \\ \boldsymbol{x}_{j}\end{array}\right]$ depending on the orientation chosen. Thus a DMU $\left(\boldsymbol{u}_{j}\right.$ $\in \mathrm{T}, j=1,2, \ldots, \mathrm{n}$ ) is efficient if and only if (iff) the optimal value $Z^{*}$ of model (2.1a) equals 0 . Then all slack variables $\boldsymbol{s}^{-}$and $\boldsymbol{s}^{+}$equal zero as well. Otherwise, the DMU is inefficient (Charnes et al. [1994]). Efficiency considerations can be made using the dual model (2.1b) as well. Note that in our formulation, $\sigma$ is zero for efficient units - not one as usually.

\subsection{DEA Model with Weights Restrictions}

The demands of real life applications probably are the origin of the incorporation of preference information in DEA. A natural incentive is some a priori knowledge on prices or the relative importance of outputs but also the need to have more realistic efficiency scores as well as the need to rank the efficient units.Because in the DEAliterature, so far only linear weights restrictions have been encountered, we may present the weights restrictions as follows:

$$
-\mu^{T} \mathbf{B}^{y}+v^{T} \mathbf{B}^{x} \geq \boldsymbol{c},
$$

where $\mathbf{B}^{y} \in \mathfrak{R}^{p \times r}$ and $\mathbf{B}^{x} \in \mathfrak{R}^{m \times r}$. Dimension $\mathrm{r}$ refers to the number of constraints.

If $c \neq 0$ we refer to them as absolute weights restrictions. The absolute weights restrictions are typically imposing a range for an individual weight. This approach was developed by Dyson and Thanassoulis [1988] and generalized by Roll et al. [1991]. Also virtual weights restrictions introduced by Wong and Beasley [1990] belong to this category.

If $\boldsymbol{c}=\boldsymbol{O}$ we speak about relative weights restrictions. The class of relative weights restrictions includes among others, the assurance region models by Thompson et al. [1990] as well as cone ratio DEA models (e.g. Charnes et al [1989]). Golany and Roll (1994) introduced standards in DEA which resulted in a model where the upper and lower bounds of the weights are treated as variables.

Roll et al. [1991] showed that the linear weights restrictions work as artificial units. This can be easily seen by adding the weights restrictions into the dual model (2.1b) and writing the primal and dual weights restricted DEA-models.

In the primal model (2.2a) new columns appear, which correspond to the new units in the problem. Note, however, that the primal's $\theta$-variables do not appear in the constraint $\mathbf{A} \lambda+\delta=\boldsymbol{b}$. Another deviation from a "standard" unit is that new inputoutput vectors may contain zero and/or negative elements. 


\begin{tabular}{|c|c|}
\hline $\begin{array}{c}\text { General DEA Model } \\
\text { with weights restrictions } \\
\text { (Primal) }\end{array}$ & $\begin{array}{c}\text { General DEA Model } \\
\text { with weights restrictions } \\
\text { (Dual) }\end{array}$ \\
\hline 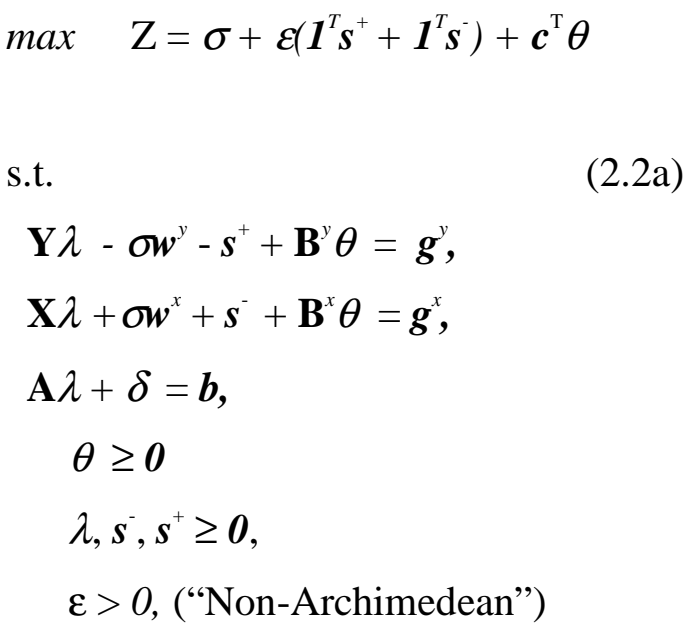 & $\begin{array}{l}\min \quad W=v^{T} \boldsymbol{g}^{x}-\mu^{T} \boldsymbol{g}^{y}+\eta^{T} \boldsymbol{b} \\
\text { s.t. } \\
\qquad \begin{array}{l}-\mu^{T} \mathbf{Y}+v^{T} \mathbf{X}+\eta^{T} \mathbf{A}-\gamma^{T}=\boldsymbol{0}^{T} \\
\mu^{T} \boldsymbol{w}^{y}+v^{T} \boldsymbol{w}^{x} \quad=1 \\
-\mu^{T} \mathbf{B}^{y}+v^{T} \mathbf{B}^{x} \geq \boldsymbol{c} \\
\mu, v \geq \varepsilon \boldsymbol{I} \\
\quad \gamma \geq \boldsymbol{0}, \eta \geq \boldsymbol{0} \\
\quad \varepsilon>0 \text { ("Non-Archimedean") }\end{array}\end{array}$ \\
\hline
\end{tabular}

Note that the weights restrictions modify $\mathrm{T}$, the set of possible input-output vectors. The original efficient frontier clearly changes after the incorporation of weights restrictions. The resulting new feasible set is $\mathrm{T}^{\mathrm{w}}=\{\boldsymbol{u} \mid \boldsymbol{u}=\mathbf{U} \boldsymbol{\lambda}+\mathbf{B} \theta, \lambda \in \Lambda, \theta \geq \boldsymbol{0}\}$. Clearly $\mathrm{T} \subseteq \mathrm{T}^{\mathrm{w}}$ . Thus the units originally efficient in set $\mathrm{T}$ are not necessarily efficient in $\mathrm{T}^{\mathrm{w}}$.

Especially, when the absolute weights restriction method is used, there is a possibility that model (2.2b) may not have a feasible solution and the primal (2.2a) is unbounded. It may, of course, happen in case of relative weights as well. In that case the weights restrictions are in conflict with one another.

\subsection{An Example - Efficiency Analysis of Municipal Dental Units}

Our data from year 1995 consists of 21 Finnish municipal dental units. The units are evaluated with three input variables: material costs (1000 FIM), dentists (working years) and other staff (working years). The two output-variables are the number of patients treated divided into two categories: less than 18 years and more than or equal to 18 years old.

We will impose the following additional weights restrictions into the model:

$$
\begin{aligned}
& v_{2} \geq 1.5 v_{3} \\
& \mu_{1} \leq \mu_{2} .
\end{aligned}
$$


The weight (the annual costs) of dentists should be at least 150 per cent of that of the other staff. Moreover the weight (cost) of younger patients should be less or equal to that of the older patients. If we did not introduce this restriction, units could manipulate their productivity by favoring the treatments of younger, easier patients.

The weights restrictions are based on information about relative prices. Note that each relative weights restriction corresponds to inputs/outputs measured in the same units.

Table 2.1 The dental units' input-output data

\begin{tabular}{|l|r|r|r|r|r|}
\hline & inputs & & & Outputs & \\
\hline Dental unit & materials & dentists & o staff & patients <18 & patients $>18$ \\
\hline Helsinki & 5648 & 132 & 212 & 73158 & 54154 \\
\hline Espoo & 2396 & 54,5 & 83 & 33098 & 25357 \\
\hline Tampere & 2789 & 40,5 & 52 & 28486 & 17157 \\
\hline Vantaa & 2231 & 59,5 & 85 & 29580 & 10624 \\
\hline Turku & 2061 & 53,5 & 52 & 24139 & 17291 \\
\hline Oulu & 1689 & 36,5 & 61,5 & 20223 & 11293 \\
\hline Lahti & 1001 & 27 & 43 & 20565 & 14251 \\
\hline Kuopio & 898 & 29,5 & 37,5 & 14049 & 9348 \\
\hline Pori & 1393 & 26,5 & 33 & 13785 & 7415 \\
\hline Jyväskylä & 973 & 23 & 34,5 & 12106 & 11945 \\
\hline Hämeenlinna & 1511 & 27,5 & 40,5 & 13247 & 9958 \\
\hline Lappeenranta & 799 & 15,5 & 21 & 10737 & 4164 \\
\hline Kotka & 523 & 16 & 27 & 10021 & 4480 \\
\hline Vaasa & 1341 & 21,5 & 29 & 10534 & 6200 \\
\hline Mikkeli & 758 & 21 & 25,5 & 10526 & 5298 \\
\hline Porvoo & 1046 & 19,5 & 30,5 & 9687 & 5349 \\
\hline Joensuu & 1296 & 22 & 29 & 8958 & 6575 \\
\hline Seinäjoki & 767 & 18 & 24 & 10161 & 9207 \\
\hline Rauma & 960 & 23 & 29,5 & 9452 & 8245 \\
\hline Kokkola & 972 & 17 & 22 & 9520 & 2753 \\
\hline Kouvola & 550 & 17 & 20,5 & 8238 & 5647 \\
\hline
\end{tabular}


Table 2.2 The combined DEA inefficiency scores without and with weights restrictions

\begin{tabular}{|l|r|r|}
\hline & \multicolumn{1}{l|}{ scores } & \\
\hline dental units & \multicolumn{1}{l|}{ combined } & with weigh \\
\hline & \multicolumn{1}{l|}{ DEA } & restrictions \\
\hline Helsinki & 0.000 & 0.000 \\
\hline Espoo & 0.000 & 0.000 \\
\hline Tampere & 0.000 & 0.000 \\
\hline Vantaa & 0.077 & 0.226 \\
\hline Turku & 0.000 & 0.098 \\
\hline Oulu & 0.139 & 0.183 \\
\hline Lahti & 0.000 & 0.000 \\
\hline Kuopio & 0.123 & 0.143 \\
\hline Pori & 0.111 & 0.177 \\
\hline Jyväskylä & 0.002 & 0.002 \\
\hline Hämeenlinna & 0.173 & 0.176 \\
\hline Lappeenranta & 0.001 & 0.106 \\
\hline Kotka & 0.035 & 0.113 \\
\hline Vaasa & 0.187 & 0.208 \\
\hline Mikkeli & 0.089 & 0.201 \\
\hline Porvoo & 0.207 & 0.247 \\
\hline Joensuu & 0.222 & 0.240 \\
\hline Seinäjoki & 0.000 & 0.000 \\
\hline Rauma & 0.149 & 0.164 \\
\hline Kokkola & 0.097 & 0.235 \\
\hline Kouvola & 0.094 & 0.159 \\
\hline
\end{tabular}

The weights restrictions result in scores that show the unit in a less favorable light. In this example, however, they did not affect the scores in any dramatic way.

\section{Setting restrictions on Multipliers in Value Efficiency Analysis}

\subsection{Value Efficiency Analysis}

The idea of Value Efficiency Analysis is to incorporate the DM's preference information into the analysis in the form of a desirable combination of inputs and outputs, not setting absolute or relative weights restrictions. As explained in Halme et al. [1998], preference information is incorporated via the Most Preferred Solution, i.e. a (virtual or existing) DMU on the efficient frontier having the best combination of of inputs and outputs. It is obvious that the MPS is efficient.

The purpose of Value Efficiency Analysis is to evaluate the efficiency of each unit in relation to the indifference contour of the Decision Maker's value function passing through the Most Preferred Solution. The evaluation could be done easily, if we explicitly knew the value function. In practice, the assumption is not realistic. Because the value function is unknown, we cannot characterize the indifference curve precisely but we have to approximate it. We do this by trying to find a region containing all 
vectors $\left[\begin{array}{l}\boldsymbol{y} \\ \boldsymbol{x}\end{array}\right] \in \mathfrak{R}^{p+m}$ surely less than or equally preferred to the most preferred unit. It is necessary to make assumptions about the value function.

Halme et al. [1998] developed the requisite theory by assuming that the DM's (unknown) value function $v(\boldsymbol{u}), \boldsymbol{u}=\left[\begin{array}{c}\boldsymbol{y} \\ \boldsymbol{x}\end{array}\right] \in \mathfrak{R}^{p+m}$ is pseudoconcave, and strictly increasing in $\boldsymbol{u}$ (i.e. strictly increasing in $\boldsymbol{y}$ and strictly decreasing in $\boldsymbol{x}$ ) and with a maximal value $v\left(\boldsymbol{u}^{*}\right), \boldsymbol{u}^{*}=\left[\begin{array}{c}\boldsymbol{y}^{*} \\ -\boldsymbol{x}^{*}\end{array}\right] \in \mathrm{T}$, at the Most Preferred Solution $\boldsymbol{u}^{*}$. Because the value function is pseudoconcave, then the region containing all vectors $\boldsymbol{u} \in \mathfrak{R}^{p+m}$ surely less than or equally preferred to the Most Preferred Unit can be characterized by the tangent hyperplanes of all possible pseudoconcave value functions obtaining their maximum at the MPS as explained in Halme et al. [1998]. Those hyperplanes define a new 'Efficiency Frontier' and efficiency is then defined in relation to this frontier using a standard DEA-technique. The resulting scores are called Value Efficiency Scores. Because we use an approximation and not the true indifference contour of the value function the resulting value efficiency scores are actually optimistic approximations of the true scores.

The basic idea of Value Efficiency Analysis is illustrated in Fig.1. We have five units $(\mathrm{A}, \mathrm{B}, \mathrm{C}, \mathrm{D}, \mathrm{E})$, which produce two outputs and use the same amount of one input. Consider the output-oriented model and illustrate the problem in the output space. Clearly all units except unit B are efficient. The classic efficiency measure for unit B is the ratio: $\frac{\mathrm{OB}}{\mathrm{OB}^{1}}$. In Value Efficiency Analysis, we would like to evaluate the ratio: $\frac{\mathrm{OB}}{\mathrm{OB}^{4}}$, but because the value function is unknown that is not possible. If we could approximate the indifference contour by a tangent, then we could use the ratio: $\frac{\mathrm{OB}}{\mathrm{OB}^{3}}$. However, we cannot assume the tangent is known. This is why we consider all possible tangents of the contour. This idea leads to the use of the ratio: $\frac{\mathrm{OB}}{\mathrm{OB}^{2}}$ as an approximate measure for Value Efficiency. Without any additional knowledge of the value function this approximation is the best we can get and we call the ratio $\frac{\mathrm{BB}^{2}}{\mathrm{OB}^{2}}$ Value Efficiency Score. Note that $\frac{\mathrm{BB}^{2}}{\mathrm{OB}^{2}}=0$ for a value efficient unit. 


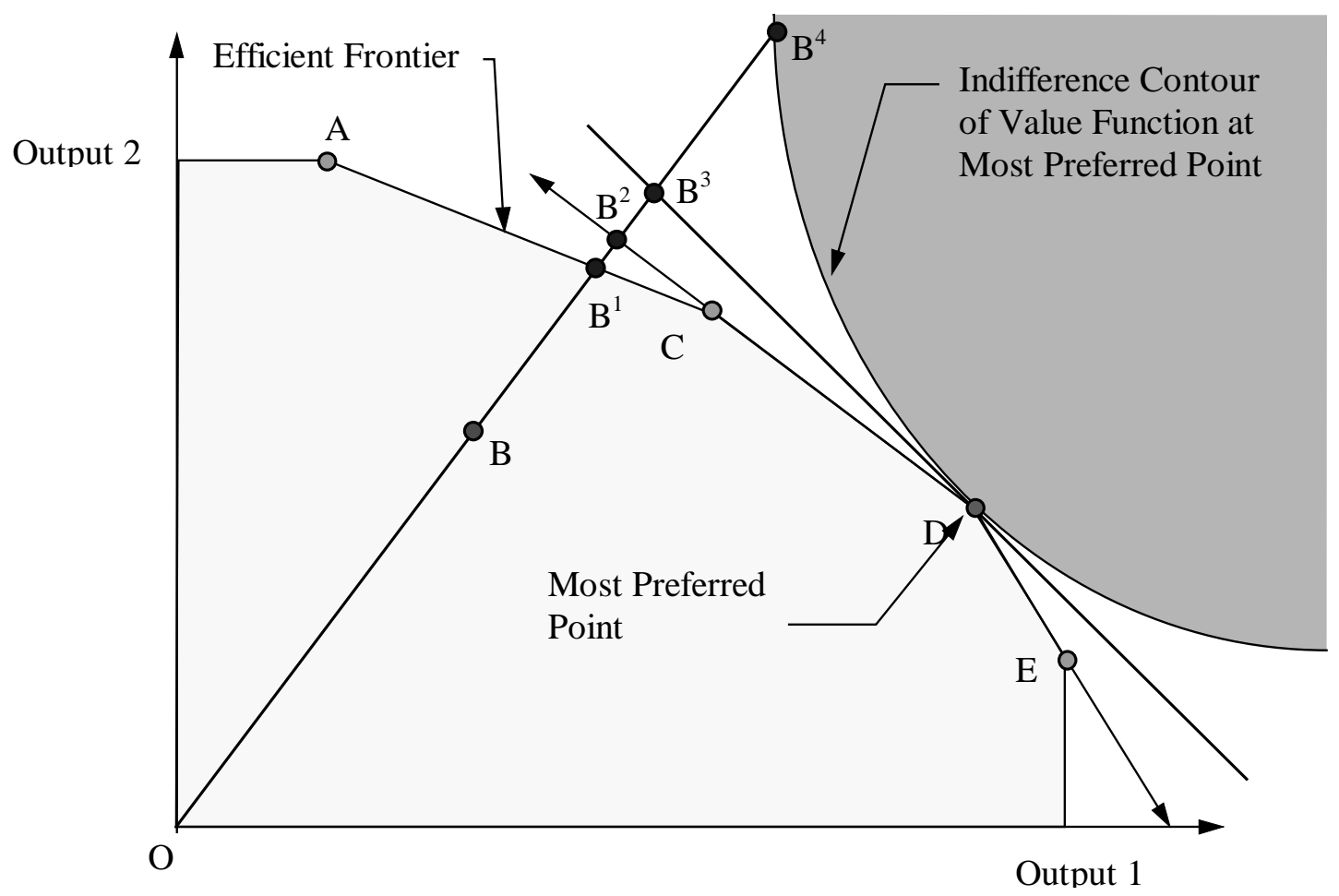

Figure 1: Illustration of Value Efficiency Analysis

Value efficiency scores can be calculated as easily as efficiency scores in standard DEA by solving a linear programming problem. $\mathrm{A} \mathrm{DMU}_{0}$ with input-output vectors $\boldsymbol{u}^{0}=\left[\begin{array}{c}\boldsymbol{y}^{0} \\ -\boldsymbol{x}^{0}\end{array}\right]$ is value inefficient with respect to any strictly increasing pseudoconcave value function $v(\boldsymbol{u}), \boldsymbol{u}=\left[\begin{array}{c}\boldsymbol{y} \\ -\boldsymbol{x}\end{array}\right]$ with a maximum at $\boldsymbol{u}^{*}$, if the optimum value $\mathrm{Z}^{*}=\mathrm{W}^{*}$ is strictly positive in this problem formulation (3.1a-b). 


\begin{tabular}{|c|c|}
\hline $\begin{array}{c}\text { General Value Efficiency Model } \\
\text { (Primal) }\end{array}$ & $\begin{array}{c}\text { General Value Efficiency Model } \\
\text { (Dual) }\end{array}$ \\
\hline 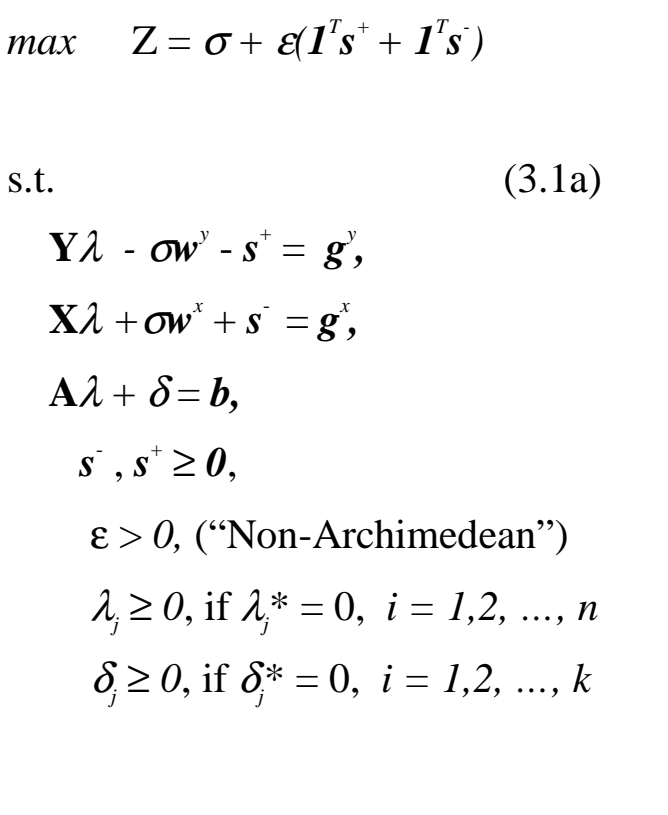 & $\begin{array}{l}\text { Min } W=v^{T} \boldsymbol{g}^{x}-\mu^{T} \boldsymbol{g}^{y}+\eta^{T} \boldsymbol{b} \\
\text { s.t. } \\
\qquad \begin{array}{l}\mu^{\mathrm{T}} \mathbf{Y}+v^{\mathrm{T}} \mathbf{X}+\eta^{\mathrm{T}} \mathbf{A}-\gamma=\mathbf{0} \\
\mu^{T} \boldsymbol{w}^{y}+v^{\mathrm{T}} \boldsymbol{w}^{x} \quad=1 \\
\mu, v \geq \varepsilon \boldsymbol{I}\end{array} \\
\gamma_{j}\left\{\begin{array}{l}\geq 0, \text { if } \lambda j^{*}=0 \\
=0, \text { if } \lambda j^{*}>0\end{array}, j=1,2, \ldots, n\right. \\
\eta_{j}\left\{\begin{array}{l}\geq 0, \text { if } \delta_{j}^{*}=0 \\
=0, \text { if } \delta_{j}^{*}>0\end{array}, j=1,2, \ldots, k\right. \\
\varepsilon>0, \text { (“Non-Archimedean”) }\end{array}$ \\
\hline
\end{tabular}

The only difference compared with standard primal DEA-models is that some variables are allowed to have negative values. This simple modification of the DEA model makes it possible to take into account value judgments in the form of the MPS.

\subsection{Restricting Weights in Value Efficiency Analysis}

A Value Efficiency Analysis model incorporates preference information into the efficiency analysis by using information about the MPS and the functional form of the value function. However, we may wish to impose additional weights restrictions, absolute or relative, in problem (3.1b). The reason for this might be the knowledge of relative prices or relative importance of some of the input-output variables. Also there might arise a need to check if the additional weights restrictions are in harmony with the MPS chosen. Thus information on the absolute or relative weights may be used as a supplement to Value Efficiency Analysis and thus to improve Value Efficiency Score estimates. Also, it may happen the DM is unable to locate a unique MPS. Then the weights restrictions may be used to choose among the MPS candidates, particularly some of them can be rejected owing to the conflict with the new information.

To impose weights restriction in Value Efficiency Analysis, we supplement the dual model (3.1b) with linear constraints: $-\mu^{T} \mathbf{b}^{y}+v^{\mathrm{T}} \mathbf{B}^{x} \geq \boldsymbol{c}, \mathbf{B}^{\mathrm{y}} \in \mathfrak{R}^{p \chi_{r}}$ and $\mathbf{B}^{x} \in \mathfrak{R}^{m \chi^{r}}$ in the same way as weights restrictions were added in the standard DEA model (2.1b). In 
(3.2a) we present the dual model with weights restrictions and the corresponding primal model with additional columns corresponding to the new restrictions.

\begin{tabular}{|c|c|}
\hline $\begin{array}{c}\text { Restricted Value Efficiency Model } \\
\text { (Dual) }\end{array}$ & $\begin{array}{c}\text { Restricted Value Efficiency Model } \\
\text { (Primal) }\end{array}$ \\
\hline $\min \quad W=v^{T} \boldsymbol{g}^{x}-\mu^{T} \boldsymbol{g}^{y}+\eta^{T} \boldsymbol{b}$ & $\operatorname{Max} \quad \mathrm{Z}=\sigma+\varepsilon\left(\boldsymbol{1}^{T} \boldsymbol{s}^{+}+\boldsymbol{I}^{T} \boldsymbol{s}^{-}\right)+\boldsymbol{c}^{T} \theta$ \\
\hline $\begin{array}{l}\text { s.t. } \\
\begin{array}{l}-\mu^{\mathrm{T}} \mathbf{Y}+v^{\mathrm{T}} \mathbf{X}+\eta^{\mathrm{T}} \mathbf{A}-\gamma=\mathbf{0} \\
\mu^{T} \boldsymbol{w}^{y}+v^{\mathrm{T}} \boldsymbol{w}^{x} \\
-\mu^{T} \mathbf{b}^{y}+v^{\mathrm{T}} \mathbf{B}^{x}-\xi \quad=c\end{array} \\
\mu, v \geq \varepsilon \boldsymbol{1} \\
\gamma_{j}\left\{\begin{array}{l}\geq 0, \text { if } \lambda j^{*}=0 \\
=0, \text { if } \lambda j^{*}>0\end{array}, j=1,2, \ldots, n\right. \\
\eta_{j}\left\{\begin{array}{l}\geq 0, \text { if } \delta_{j}^{*}=0 \\
=0, \text { if } \delta_{j}^{*}>0\end{array}, j=1,2, \ldots, k\right. \\
\xi \geq \boldsymbol{0} \\
\varepsilon>0, \text { (“Non-Archimedean”) }\end{array}$ & 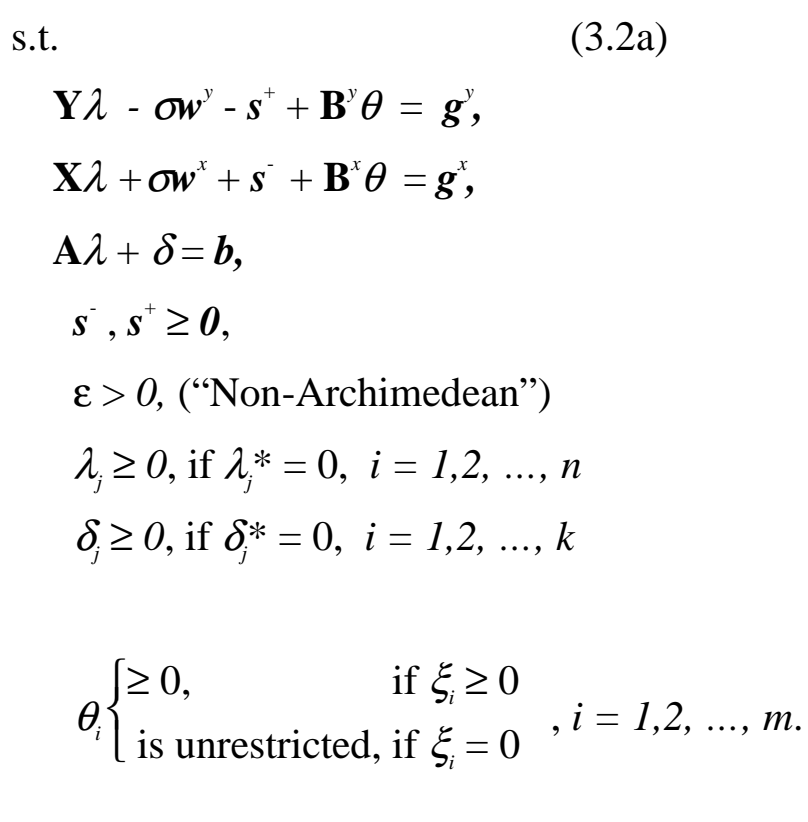 \\
\hline and $\delta^{*}$ & ution: $y^{*}=$ \\
\hline
\end{tabular}

Problems (3.1a-b) always have a (finite) solution (if 2.1a-b has a (finite) solution and) if the MPS is efficient. However, it may occur that the dual (3.2b) has no feasible solution and the primal's optimum is unbounded. It means that the weights constraints $-\mu^{T} \mathbf{b}^{y}+$ $v^{\mathrm{T}} \mathbf{B}^{x} \geq \boldsymbol{c}$ are in conflict with the MPS or it may happen that after inserting them to the basic model (2.1b), model (2.2b) have no feasible solution.

If the problem of infeasibility only appears in model (3.2b), but not in model (2.3b) we may project the MPS onto the efficient frontier of the weights restricted production set $\mathrm{T}^{\mathrm{w}}$. This can be done by solving (2.2a) or (2.2b) with the MPS as the unit zero. This resulting point on the efficient frontier is then used as a new MPS. Alternatively, we may give the DM an opportunity to consider his MPS choice and possibly find another one, It may be possible that the conflict also appears in the original models $(2.2 \mathrm{a}, \mathrm{b})$. In that case we have to ask the DM to re-consider the weights restrictions. The main end is to have an MPS in harmony with the additional weights restrictions.

Alternatively, we may start readily from the model (2.2a) and then search the efficient frontier of set $\mathrm{T}^{\mathrm{W}}$. An advantage of this approach is that we immediately see if the 
weights restrictions are in conflict with the original model. A drawback is that we cannot guarantee that the MPS found belongs to the feasible set of the original problem (2.1a). In this case, we may consider the MPS as a virtual unit which may lie on the "true" efficient frontier, but not on the estimated frontier. Anyhow, we want to locate the most preferred unit on the modified efficient frontier, also belonging to the original efficient frontier. After finding that we may carry out Value Efficiency Analysis.

It is up to the DM and may depend on the problem, in which order the DM likes to carry out the two phases: set up weight restrictions and locate the MPS. In each case, Value Efficiency Analysis is carried out in a straightforward way with model (3.2a) or (3.2b). In our presentation we prefer to use the primal model (3.2a).

\subsection{An Example - Value Efficiency Analysis of Municipal Dental Units}

Consider further the municipal dental units. We carry out Value Efficiency Analysis first without weights restrictions and then with the restrictions used in Subsection 2.3:

$$
\begin{aligned}
& v_{2} \geq 1.5 v_{3} \\
& \mu_{1} \leq \mu_{2} .
\end{aligned}
$$

Assume that the DM names Espoo as the most preferred solution. In this case, we may think that the DM considers it as an example unit preferred to all others. The corresponding value inefficiency scores obtained with the combined model (3.1a) are reported in Table 3.1 .

The analysis is repeated by imposing the weights restrictions and using (3.2b). The results are also reported in Table 3.1. We see that the scores for most of the units are strictly less optimistic than the Value Efficiency Scores without weights restrictions in the second column.

As we already discussed above, an efficient unit in the original set $\mathrm{T}$ is not necessarily efficient in set $\mathrm{T}^{\mathrm{w}}$. Consider e.g. unit Turku as the most preferred solution. After imposing the weights restrictions and trying to solve the model (3.2b) for any unit, the solution is unbounded. It means that Turku is not on the efficient frontier in production set $\mathrm{T}^{\mathrm{w}}$. One solution is to first project Turku onto the efficient frontier by model (2.2a), and then use this point as the most preferred solution in model (3.1b), or we may ask the DM to try another most preferred solution. 
Table 3.1. Value Inefficiency and restricted Value Inefficiency Scores

\begin{tabular}{|l|r|r|r|}
\hline & \multicolumn{1}{|l|}{ combined } & value eff & \multicolumn{1}{l|}{ value eff } \\
\hline & DEA & MPS Espoo & weight restr \\
\hline Helsinki & 0.000 & 0.000 & 0.000 \\
\hline Espoo & 0.000 & 0.000 & 0.000 \\
\hline Tampere & 0.000 & 0.000 & 0.000 \\
\hline Vantaa & 0.072 & 0.143 & 0.215 \\
\hline Turku & 0.000 & 0.000 & 0.098 \\
\hline Oulu & 0.122 & 0.161 & 0.192 \\
\hline Lahti & 0.000 & 0.000 & 0.000 \\
\hline Kuopio & 0.110 & 0.150 & 0.227 \\
\hline Pori & 0.100 & 0.201 & 0.270 \\
\hline Jyväskylä & 0.002 & 0.002 & 0.015 \\
\hline Hämeenlinna & 0.148 & 0.150 & 0.192 \\
\hline Lappeenranta & 0.001 & 0.238 & 0.331 \\
\hline Kotka & 0.034 & 0.272 & 0.379 \\
\hline Vaasa & 0.158 & 0.235 & 0.340 \\
\hline Mikkeli & 0.081 & 0.247 & 0.363 \\
\hline Porvoo & 0.172 & 0.286 & 0.407 \\
\hline Joensuu & 0.181 & 0.216 & 0.315 \\
\hline Seinäjoki & 0.000 & 0.000 & 0.037 \\
\hline Rauma & 0.130 & 0.132 & 0.197 \\
\hline Kokkola & 0.089 & 0.316 & 0.489 \\
\hline Kouvola & 0.086 & 0.184 & 0.291 \\
\hline
\end{tabular}

\section{Further Considerations}

\subsection{The set of weights, optimal for an efficient unit}

In some problems, the DM may be unable to set explicit restrictions for weights, but instead he/she may be willing to consider which weights are in harmony with a certain $\operatorname{MPS}\left[\begin{array}{l}\boldsymbol{y}^{*} \\ \boldsymbol{x}^{*}\end{array}\right]=\left[\begin{array}{l}\mathbf{Y} \\ \mathbf{X}\end{array}\right] \lambda^{*}$. The optimal solutions of the dual $(2.1 \mathrm{~b})$, where vector $\boldsymbol{g}=\left[\begin{array}{l}\boldsymbol{g}^{\boldsymbol{y}} \\ \boldsymbol{g}^{\boldsymbol{x}}\end{array}\right]$ refers to the MPS, can be found considering the optimality conditions (see, e.g. Bazaraa, Jarvis, and Sherali [1990]) of the primal (2.1a).

First, permute and decompose the columns of matrices $\mathbf{X}, \mathbf{Y}$, and $\mathbf{A}$ such that $\mathbf{X} \rightarrow\left[\mathbf{X}_{\mathrm{B}}\right.$ $\left.\mathbf{X}_{\mathrm{N}}\right], \mathbf{Y} \rightarrow\left[\begin{array}{ll}\mathbf{Y}_{\mathrm{B}} & \mathbf{Y}_{\mathrm{N}}\end{array}\right]$, and $\mathbf{A} \rightarrow\left[\begin{array}{ll}\mathbf{A}_{\mathrm{B}} & \mathbf{A}_{\mathrm{N}}\end{array}\right]$, where subscript 'B' refers to the columns corresponding to values $\lambda_{j}^{*}>0, j \in\{1,2, \ldots, \mathrm{n}\}$ and ' $\mathrm{N}$ ' to the remaining columns $\lambda_{j}^{*}=$ $0, j \in\{1,2, \ldots, \mathrm{n}\}$. Decompose further $\gamma=\left[\begin{array}{l}0 \\ \gamma_{\mathrm{N}}\end{array}\right] \in \mathfrak{R}^{p}$ and $\eta=\left[\begin{array}{l}\boldsymbol{0} \\ \eta_{\mathrm{N}}\end{array}\right] \in \mathfrak{R}^{k}$ as well as the vector $\boldsymbol{b}=\left[\begin{array}{c}\boldsymbol{0} \\ \boldsymbol{b}_{\mathrm{N}}\end{array}\right]$ and the rows of the matrix $\mathbf{A}=\left[\begin{array}{ll}\mathbf{A}_{\mathrm{B}} & \mathbf{A}_{\mathrm{N}}\end{array}\right]$ into $\mathbf{A}=\left[\begin{array}{ll}\mathbf{A}_{\mathrm{BB}} & \mathbf{A}_{\mathrm{NB}} \\ \mathbf{A}_{\mathrm{BN}} & \mathbf{A}_{\mathrm{NN}}\end{array}\right]$, where subscript 'B' refers to the columns corresponding to values $\delta_{j}^{*}>0, j \in\{1,2, \ldots$, $\mathrm{k}\}$.

Now the optimality conditions for a MPS can be written as follows: 


$$
\begin{aligned}
& -\mu^{\mathrm{T}} \mathbf{Y}_{\mathrm{B}}+v^{\mathrm{T}} \mathbf{X}_{\mathrm{B}}+\eta_{\mathrm{N}_{\mathrm{BN}}^{\mathrm{T}}}^{\mathrm{A}}=\boldsymbol{0}^{T} \\
& -\mu^{\mathrm{T}} \mathbf{Y}_{\mathrm{N}}+v^{\mathrm{T}} \mathbf{X}_{\mathrm{N}}+\eta_{\mathrm{N}}^{\mathrm{T}} \mathbf{A}_{\mathrm{NN}}-\gamma_{\mathrm{N}}=\boldsymbol{0}^{T} \\
& \mu^{T} \boldsymbol{w}^{y}+v^{\mathrm{T}} \boldsymbol{w}^{x}=1 \\
& \mu, v \geq \varepsilon \boldsymbol{1}, \eta_{\mathrm{N}} \geq \boldsymbol{0} \\
& \boldsymbol{\varepsilon}>0 .
\end{aligned}
$$

The set of optimal weights corresponding to the MPS is a convex polyhedron. By enumerating all possible extreme point solutions of (4.1), we will find a representation for all possible weights. As an enumeration scheme one may use e.g. the algorithm of Dyer et al [1977].

Denote those solutions by $\left[\begin{array}{c}\mu^{\mathrm{i}} \\ v^{\mathrm{j}}\end{array}\right], i \in \mathrm{M}$, where $\mathrm{M}$ consists of the indices of all extreme point solutions. The possible weights vectors $\pi$ corresponding to the MPS selected are then of the form

$$
\pi=\sum_{i \in M} \alpha_{i}\left[\begin{array}{c}
\mu^{i} \\
v^{i}
\end{array}\right], \boldsymbol{I}^{T} \alpha=1 .
$$

The set of possible weights is not necessary small. In that case, it is not necessary easy to enumerate possible weights. It means that this approach can be used only when enumeration is easy to be performed. The equation system (4.1) can be used to check the consistency of additional weights restrictions. In that case, enumeration is not needed. We simply augment the equation system (4.1) with additional constraints, and will check if the system has feasible solutions.

\subsection{Illustrative Example}

Consider a simple example of five units with one input and two outputs.

Table 4.1: Data Set for Illustrative Example

\begin{tabular}{|c|c|c|c|}
\hline Units & Input & Output $_{1}$ & Output $_{2}$ \\
\hline $\mathrm{A}$ & 1 & 3 & 2 \\
\hline $\mathrm{B}$ & 4 & 3 & 3 \\
\hline $\mathrm{C}$ & 7 & 5 & 2 \\
\hline $\mathrm{D}$ & 13 & 7 & 3 \\
\hline $\mathrm{E}$ & 12 & 11 & 2 \\
\hline
\end{tabular}


Consider the combined BCC model. Units A, B, D and E are efficient. Assume that the DM will locate the point $(5.84,5.80,2.33)$ as his/her MPS. The MPS is obtained by combining the values of units $\mathrm{A}, \mathrm{B}$ and $\mathrm{E}$ using the coefficients $(0.32,0.33,0.35)$ correspondingly. Thus $\lambda_{A}>0, \lambda_{B}>0, \lambda_{C}=0, \lambda_{D}=0, \lambda_{E}>0$, and $\delta=0$. Thus our equation system is:
A: $\quad v_{1}-3 \mu_{1}-2 \mu_{2}+\eta=0$
B: $\quad 4 v_{1}-3 \mu_{1}-3 \mu_{2}+\eta=0$
C: $\quad 7 v_{1}-5 \mu_{1}-2 \mu_{2}+\eta \geq 0$
D: $\quad 13 v_{1}-7 \mu_{1}-3 \mu_{2}+\eta \geq 0$
E: $\quad 12 \nu_{1}-11 \mu_{1}-2 \mu_{2}+\eta=0$
$7 v_{1}+5 \mu_{1}+2 \mu_{2}=1$
$\mu, v \geq 0$

Note that we use the last row in this form for simplicity. We have four equalities, two inequalities and four variables, so the solution is unique, because the rows (columns) of those four equalities are linearly independent. It is $\mathbf{v}^{1}=\left(v_{1}, \mu_{1}, \mu_{2}, \eta\right)=(0.050,0.069$, $0.151,0.459)$.

Let's consider another example, where the MPS $(2.5,3,2.5)$ is found by combining A and $\mathrm{B}$ using the coefficients $(0.5,0.5)$. In this case, the equation system is of the form:
A: $\quad v_{1}-3 \mu_{1}-2 \mu_{2}+\eta=0$
B: $\quad 4 v_{1}-3 \mu_{1}-3 \mu_{2}+\eta=0$
C: $\quad 7 v_{1}-5 \mu_{1}-2 \mu_{2}+\eta \geq 0$
D: $\quad 13 v_{1}-7 \mu_{1}-3 \mu_{2}+\eta \geq 0$
E: $\quad 12 \nu_{1}-11 \mu_{1}-2 \mu_{2}+\eta \geq 0$

$$
\begin{aligned}
& 7 v_{1}+5 \mu_{1}+2 \mu_{2}=1 \\
& \mu, v \geq 0 .
\end{aligned}
$$

The solution set is the convex combination of two extreme points, $\mathbf{v}^{1}=(0.05,0.069$, $0.151,0.459)$ and $\mathbf{v}^{2}=(0.077,0,0.231,0.385)$.

Finally, assume D is the MPS. The equation system looks like as follows:
A: $\quad v_{1}-3 \mu_{1}-2 \mu_{2}+\eta \geq 0$
B: $\quad 4 v_{1}-3 \mu_{1}-3 \mu_{2}+\eta \geq 0$
C: $\quad 7 v_{1}-5 \mu_{1}-2 \mu_{2}+\eta \geq 0$
D: $\quad 13 v_{1}-7 \mu_{1}-3 \mu_{2}+\eta=0$
E: $\quad 12 v_{1}-11 \mu_{1}-2 \mu_{2}+\eta \geq 0$
$7 \nu_{1}+5 \mu_{1}+2 \mu_{2}=1$
$\mu, v \geq 0$ 
The extreme points whose convex combination forms the set of solutions are $\mathbf{v}^{3}=$ $(0.026,0.059,0.261,0.856), \mathbf{v}^{4}=(0,0.077,0.308,1.461)$ and $\mathbf{v}^{5}=(0,0,0.5,1.5)$.

Let's demonstrate how to check the reasonability of some constraints. For instance, assume that $D$ is the MPS, and if we would like to add the constraint $\mu_{1} \geq \mu_{2}$. It means that the inequality is added to the last example above. We cannot find a feasible solution for the equation system. This fact can also be seen by considering the extreme point solutions as well.. In all v-vectors, $\mu_{2}>\mu_{1}$, implying that it is also true to all convex combinations as well. This approach helps us to find a consistent set of weights or reveal that our assumptions concerning the MPS and/or the value function are invalid.

\section{Conclusion}

In this paper, we have consider two approaches to incorporate preference information into Data Envelopment Analysis. Those are Value Efficiency Analysis and the use of weights restrictions. We have further shown that these approaches can be used as supplements to each other. We have illustrated the use of the approach with a case study consisting of 21 Finnish municipal dental units. The results demonstrate that the approaches can be used successfully together.

\section{References}

Allen, R., Athanassopoulos, A., Dyson, R.G. and Thanassoulis, E. (1997), "Weights Restrictions and Value Judgements in Data Envelopment Analysis: Evolution, Development and Future Directions", Annals of Operations Research 73, 13-34.

Banker, R.D., A. Charnes, and W.W. Cooper (1984), "Some Models for Estimating Technical and Scale Inefficiencies in Data Envelopment Analysis", Management Science 30, 1078-1092.

Charnes, A., Cooper, W. and Rhodes, E. (1978), "Measuring Efficiency of Decision Making Units", European Journal of Operational Research 2, 429-444.

Charnes, A., Cooper, W., Wei, Q., and Huang, Z. (1989), "Cone Ratio Data Envelopment Analysis and Multi-Objective Programming", International Journal of Systems Science 20, 1099-1118.

Charnes A., W. Cooper, A. Lewin, and L. Seiford (1994), Data Envelopment Analysis: Theory, Methodology and Applications, Kluwer Academic Publishers, Norwell.

Dyer, M. E. and Proll, L.G. (1977), “An Algorithm for Extreme Points”, Mathematical Programming 14, 249-261.

Dyson, R.G. and E. Thanassoulis (1988), "Reducing Weight Flexibility in Data Envelopment Analysis", Journal of Operational Research Society 6, 563-576.

Golany, B. (1988), “An Interactive MOLP Procedure for the Extension of DEA to Effectiveness Analysis", Journal of Operational Research Society 39, 725-734.

Golany, B and Roll, Y. (1994) "Incorporating Standards via DEA", in A. Charnes, W.W. Cooper, A.Y. Lewin, L.M. Seiford (Eds), Data Envelopment Analysis: Theory, Methodology and Applications, Kluwer Academic Publishers, Norwell. 
Halme, M., Joro, T, Korhonen, P., Salo, S. and Wallenius, J. (1998) "A Value Efficiency Approach to Incorporating Preference Information in Data Envelopment Analysis", Forthcoming in Management Science.

Joro, T., Korhonen, P. and Wallenius, J. (1998): "Structural Comparison of Data Envelopment Analysis and Multiple Objective Linear Programming", Management Science 44, 962-970.

Pedraja-Chaparro, F., Salinas-Jimenez, J. and Smith, P. (1997) "On the Role of Weight Restrictions in Data Envelopment Analysis", Journal of Productivity Analysis 8, 215-230.

Roll, Y., Cook, W.D. and Golany, B. (1991), "Controlling Factor Weights in Data Envelopment Analysis", IIE Transactions 23, 2-9.

Thanassoulis E. and R.G. Dyson (1992), "Estimating Preferred Target Input-Output Levels Using Data Envelopment Analysis", European Journal of Operational Research $56,80-97$.

Thanassoulis, E. and Allen, R. (1998), "Simulating Weights Restrictions in Data Envelopment Analysis", Management Science 44, 586-594.

Thompson, R.G., Singleton, F., Thrall, R. and Smith, B. (1986) "Comparative Site Evaluations for Locating a High-Energy Physics Lab in Texas”, Interfaces 16, 35-49.

Thompson R.G., L.M. Langemeier, C-T. Lee, E. Lee, and R.M. Thrall (1990), “Te Role of Multiplier Bounds in Efficiency Analysis with Application to Kansas Farming", Journal of Econometrics 46, 93-108

Wong Y-H.B. and J.E. Beasley (1990), "Restricting Weight Flexibility in Data Envelopment Analysis", Journal of Operational Research Society 41, 829-835. 